\title{
The Usefulness of the Measurement of Esophagogastric Junction Distensibility by EndoFLIP in the Diagnosis of Gastroesophageal Reflux Disease
}

\author{
Jung Min Lee ${ }^{1}$, In Kyung Yoo², Eunju Kim², Sung Pyo Hong², and Joo Young Cho² \\ ${ }^{1}$ Department of Gastroenterology, Wonkwang University Sanbon Medical Center, Gunpo, and 2Department of Gastroenterology, CHA \\ Bundang Medical Center, CHA University College of Medicine, Seongnam, Korea
}

\section{Article Info}

Received March 31, 2020

Revised July 22, 2020

Accepted July 29, 2020

Published online November 2, 2020

\section{Corresponding Author}

\section{Joo Young Cho}

ORCID https://orcid.org/0000-0002-7182-5806

E-mail cjy6695@naver.com

Jung Min Lee and In Kyung Yoo contributed equally to this work as first authors.

\begin{abstract}
Background/Aims: Increased esophagogastric junction (EGJ) relaxation is the most important mechanism involved in gastroesophageal reflux disease (GERD). An endoscopic functional luminal imaging probe (EndoFLIP ${ }^{\circledR}$ ) is a device used to quantify EGJ distensibility in routine endoscopy. The aim of the current study was to assess the usefulness of EndoFLIP ${ }^{\circledR}$ for the diagnosis of GERD compared to normal controls.

Methods: We analyzed EndoFLIP ${ }^{\circledR}$ data from 204 patients with erosive reflux disease (ERD), 310 patients with nonerosive reflux disease (NERD), and 277 normal subjects. EndoFLIP ${ }^{\circledR}$ uses impedance planimetry to measure 16 cross-sectional areas (CSAs) in conjunction with the corresponding intrabag pressure within a $4.6 \mathrm{~cm}$ cylindrical segment of a fluid-filled bag. The EGJ distensibility was assessed using $40 \mathrm{~mL}$ volume-controlled distensions.

Results: The mean distensibility index values were $13.98 \mathrm{~mm}^{2} / \mathrm{mm} \mathrm{Hg}$ in ERD patients, 11.42 $\mathrm{mm}^{2} / \mathrm{mm} \mathrm{Hg}$ in NERD patients, and $9.1 \mathrm{~mm}^{2} / \mathrm{mm} \mathrm{Hg}$ in normal subjects. There were significant differences in EGJ distensibility among the three groups $(p<0.001)$. In addition, the CSAs were significantly higher in the ERD $\left(291.03 \pm 160.77 \mathrm{~mm}^{2}\right)$ and NERD groups $\left(285.87 \pm 155.47 \mathrm{~mm}^{2}\right)$ than in the control group $\left(249.78 \pm 144.76 \mathrm{~mm}^{2}, \mathrm{p}=0.004\right)$. We determined the distensibility index cutoff value of EGJ as 10.95 for the diagnosis of GERD by receiver operating characteristic curve analysis.

Conclusions: The EGJ distensibilities of GERD patients were higher than those of normal subjects, regardless of the presence of reflux esophagitis. Thus, the measurement of EGJ distensibility using the EndoFLIP ${ }^{\circledR}$ system could be useful in the diagnosis of GERD. (Gut Liver 2021;15:546-552)
\end{abstract}

Key Words: Gastroesophageal reflux disease; Esophagogastric junction; Functional lumen imaging probe

\section{INTRODUCTION}

Gastroesophageal reflux disease (GERD), including acid reflux and heartburn, is common worldwide. ${ }^{1}$ It is classified as erosive reflux disease (ERD) in the esophagus, and nonerosive reflux disease (NERD) without esophageal mucosal abnormality, but with symptoms such as heartburn. ${ }^{2,3}$ Increased esophagogastric junction (EGJ) relaxation is the most important pathophysiology of GERD. ${ }^{4}$ Endoscopy, high-resolution manometry, and 24-hour ambulatory $\mathrm{pH}$ monitoring all have limitations when it comes to evaluating the function of the EGJ., ${ }^{5,6}$

The endoscopic functional luminal imaging probe (EndoFLIP ${ }^{\oplus}$ ) is a medical device designed to evaluate the distensibility of the EGJ, and has been widely used for measuring esophageal functional disease in Western countries. ${ }^{7,8}$ Since the pathology of GERD involves reflux occurring when the distensibility of the EGJ increases, 
EndoFLIP $^{\circledast}$ is useful in the diagnosis of GERD., ${ }^{9,10}$ A previous small-population study showed that EndoFLIP ${ }^{\oplus}$ helps to evaluate GERD by evaluating the function of the EGJ. ${ }^{8}$ However, another study demonstrated controversies surrounding the usefulness of EndoFLIP ${ }^{\circledast}$ in GERD patients. ${ }^{11}$

In this study, we aimed to evaluate the clinical usefulness of the measurement of EGJ distensibility using EndoFLIP $^{\oplus}$ in a large population including patients with GERD compared to control patients.

\section{MATERIALS AND METHODS}

\section{Study design}

This single-center prospective study was performed in the Department of Gastroenterology and Digestive Endoscopy at the CHA Bundang Medical Center (Seongnam, Korea) from March 2016 to October 2018. All patients provided written informed consent, and the study was approved by the Institutional Review Board of the CHA Bundang Medical Center (IRB number: CHAMC 2016-04016-013).

\section{Patients}

Individuals between 20 and 79 years of age, with typical reflux-related symptoms such as heartburn and regurgitation, and scheduled to undergo a screening gastroscopy and EndoFLIP ${ }^{\circledast}$ imaging before taking anti-acid medication, were included as GERD group in this study. Additionally, according to the results, patients with erosive esophagitis, as determined by gastroscopy, were referred to as the ERD group. Patients with no erosive esophagitis, but with typical reflux symptom and positive response to proton pump inhibitors were classified as the NERD group. ${ }^{12}$ Control group was defined as participants having nonspecific abdominal symptom, but without any typical reflux symptoms and demonstrating normal 24-hour ambulatory $\mathrm{pH}$ monitoring.

Patients were excluded for the following reasons: (1) inability to receive a gastroscopy or EndoFLIP ${ }^{\circledast}$ imaging; (2) inability to provide informed consent; (3) hemodynamic instability (systolic blood pressure $<90 \mathrm{~mm} \mathrm{Hg}$ ), or if the peripheral oxygen saturation $\left(\mathrm{SpO}_{2}\right)$ was $90 \%$ in room air, or $<95 \%$ on $2 \mathrm{~L} / \mathrm{min}$ of oxygen at baseline before the procedure; (4) psychological problems; (5) acute severe infection; and (6) evidence of esophageal motility disorder on esophageal manometry.

\section{Endoluminal functional lumen imaging probe}

EndoFLIP $^{\circledast}$ is a device used to measure EGJ distensibility. The catheter (EF-325) had a total length of $240 \mathrm{~cm}$ and an outer diameter of $3 \mathrm{~mm}$. The EndoFLIP ${ }^{\circledR}$ catheter, with 16 paired impedance electrodes and a pressure sensor, had a complaint bag at the distal end that was able to extend to $40 \mathrm{~mL}$ in volume. ${ }^{13}$ The bag was filled with the conduct medium, and the impedance electrodes were able to measure the cross-sectional area (CSA) and the response of the pressure to the increased area, defined as the distensibility. When the catheter is placed in the EGJ with the gastroscope, the activated electrodes face each other, allowing the CSA to be measured in the range of 10 to $450 \mathrm{~mm}^{2} .^{24,15}$

\section{Protocol}

All patients with any abdominal symptoms underwent gastroscopy, ambulatory $\mathrm{pH}$ monitoring, high-resolution manometry, and EndoFLIP ${ }^{\oplus}$. We conducted the studies as following order: (1) gastroscopy, (2) EndoFLIP ${ }^{\circledast}$, (3) high-resolution manometry, or (4) 24-hour monitoring on the same day. The catheter of 24-hour monitoring was removed the next day. Patients with GERD grade LA classification $\mathrm{A}$ to $\mathrm{D}$, as determined by gastroscopy, were classified into the ERD group. ${ }^{16}$ In addition, among the patients without erosion, determined by gastroscopy, patients with typical reflux symptom and response to proton pump inhibitors regardless of the results 24-hour ambulatory $\mathrm{pH}$ monitoring were classified as the NERD group. Patients who did not meet either the ERD or NERD criteria following both gastroscopy and 24-hour ambulatory $\mathrm{pH}$ monitoring, and showed normal findings, but having nonspecific abdominal symptoms were classified as the control group.

The primary outcome was the CSA and distensibility index $\left(\mathrm{mm}^{2} / \mathrm{mm} \mathrm{Hg}\right)$ measured using EndoFLIP ${ }^{\oplus}$. The measurement was taken by a physician (I.K.Y) by filling the EndoFLIP ${ }^{\oplus}$ catheter with $40 \mathrm{~mL}$ of saline and obtaining the average of three measurements.

\section{Statistical analysis}

Continuous variables are expressed as means \pm standard deviations, and discontinuous variables are expressed as counts and percentages. SPSS version 24.0 for Windows (IBM Corp., Armonk, NY, USA) was used for data entry and statistical analyses. For analyses between the groups, analysis of variance was used to compare continuous variables as appropriate, and either the chi-square test or Fisher exact test was used for categorical data. The empirical receiver operating characteristic curve, which is a plot of true positive rate versus false positive rate for all possible cutoff values, was used. Any p-values $<0.05$ were considered statistically significant. 


\section{RESULTS}

\section{Baseline characteristics}

Initially, 862 patients were assessed; among them, 49 patients were excluded because they were unable to undergo gastroscopy or EndoFLIP ${ }^{\oplus}$ and had evidence of abnormal esophageal motility disorder following esophageal manometry $(n=13)$, hemodynamic instability during the procedure $(n=5)$, severe infection $(n=3)$, or psychological problems ( $\mathrm{n}=1)$ (Fig. 1). In total, 791 patients (ERD, 204; NERD, 310; control, 277) were included in the final analysis. Across the three groups, the baseline characteristics were not significantly different in terms of age, sex, body mass index, or GERD-Q score (Table 1). Furthermore, the ERD group (6.33 \pm 8.72 years) had a significantly longer duration of symptoms compared to the other groups (NERD group, $3.24 \pm 4.04$ years; control group, $4.86 \pm 5.71$ years; $\mathrm{p}=0.021)$. In the ERD group, the LA classifications $\mathrm{A}, \mathrm{B}, \mathrm{C}$, and $\mathrm{D}$ of patients were 113 (55\%), 63 (31\%), 27 (13\%), and one (1\%), respectively.

\section{Assessment of distensibility index and CSA using EndoFLIP ${ }^{\circledR}$}

Fig. 2 shows the typical images of EndoFLIP ${ }^{\circledR}$ in the ERD and control patient. The distensibility index, measured using EndoFLIP ${ }^{\oplus}$, was significantly higher in the $\operatorname{ERD}\left(13.98 \pm 11.54 \mathrm{~mm}^{2} / \mathrm{mm} \mathrm{Hg}\right)$ and NERD $\left(11.42 \pm 9.62 \mathrm{~mm}^{2} /\right.$ $\mathrm{mm} \mathrm{Hg}$ ) groups than in the control group $\left(9.10 \pm 7.23 \mathrm{~mm}^{2} /\right.$ $\mathrm{mm} \mathrm{Hg}, \mathrm{p}<0.001$ ) (Fig. 3). In addition, the CSA was also significantly higher in the ERD $\left(291.03 \pm 160.77 \mathrm{~mm}^{2}\right)$ and NERD $\left(285.87 \pm 155.47 \mathrm{~mm}^{2}\right)$ groups than in the control group $\left(249.78 \pm 144.76 \mathrm{~mm}^{2}, \mathrm{p}=0.004\right)$ (Table 2 ).

In high-resolution manometry, the integrated relaxation pressure was significantly lower in the ERD group than in the other groups $(10.19 \pm 6.84 \mathrm{~mm} \mathrm{Hg}, \mathrm{p}=0.023)$. In the 24-hour ambulatory $\mathrm{pH}$ monitoring, both the ERD and NERD groups had a significantly higher number of reflux episodes ( $\mathrm{p}=0.044$ ), including the number of acid reflux episodes ( $\mathrm{p}=0.023)$ and nonacid reflux episodes $(\mathrm{p}=0.021)$, and DeMeester scores $(\mathrm{p}<0.001)$.

\section{Assessment of the optimal distensibility index cutoff value}

We determined the distensibility index cutoff value of

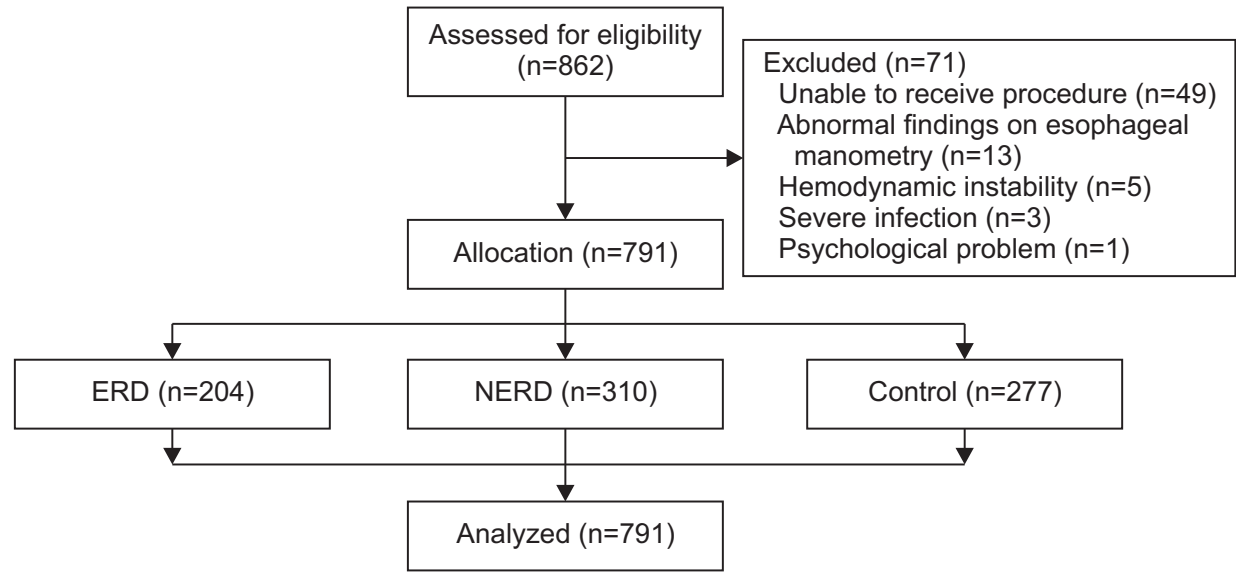

Fig. 1. Assembly of patients. Flowchart showing the recruitment of study patients.

ERD, erosive reflux disease; NERD, nonerosive reflux disease.

Table 1. Baseline Characteristics

\begin{tabular}{lcccc}
\hline \multicolumn{1}{c}{ Variable } & ERD $(\mathrm{n}=204)$ & NERD $(\mathrm{n}=310)$ & Control $(\mathrm{n}=277)$ & $\mathrm{p}$-value \\
\hline Age, $\mathrm{yr}$ & $46.53 \pm 14.50$ & $46.93 \pm 14.42$ & $49.14 \pm 15.39$ & $0.239 *$ \\
Sex (male:female) & $118: 86$ & $151: 159$ & $140: 137$ & $0.121^{\dagger}$ \\
$\mathrm{BMI}, \mathrm{kg} / \mathrm{m}^{2}$ & $22.56 \pm 3.54$ & $22.60 \pm 3.63$ & $22.31 \pm 2.78$ & $0.838^{*}$ \\
Height, $\mathrm{cm}$ & $165.70 \pm 10.41$ & $163.62 \pm 8.56$ & $164.76 \pm 10.02$ & $0.326^{*}$ \\
Weight, $\mathrm{kg}$ & $62.69 \pm 14.77$ & $61.10 \pm 12.37$ & $61.09 \pm 11.67$ & $0.678^{*}$ \\
Duration of symptom, yr & $6.33 \pm 8.72$ & $3.24 \pm 4.04$ & $4.86 \pm 5.71$ & $0.021^{*}$ \\
GERD-Q score & $8.26 \pm 2.99$ & $8.49 \pm 3.00$ & $8.19 \pm 2.72$ & $0.772^{*}$ \\
LA classification, A/B/C/D & $113(55) / 63(31) / 27(13) / 1(1)$ & & & \\
\hline
\end{tabular}

Data are presented as mean \pm SD or number $(\%)$.

ERD, erosive reflux disease; NERD, nonerosive reflux disease; BMI, body mass index; GERD-Q, gastroesophageal reflux disease questionnaire. Statistical significance between groups was tested by *analysis of variance, ${ }^{\dagger}$ chi-square analysis. 


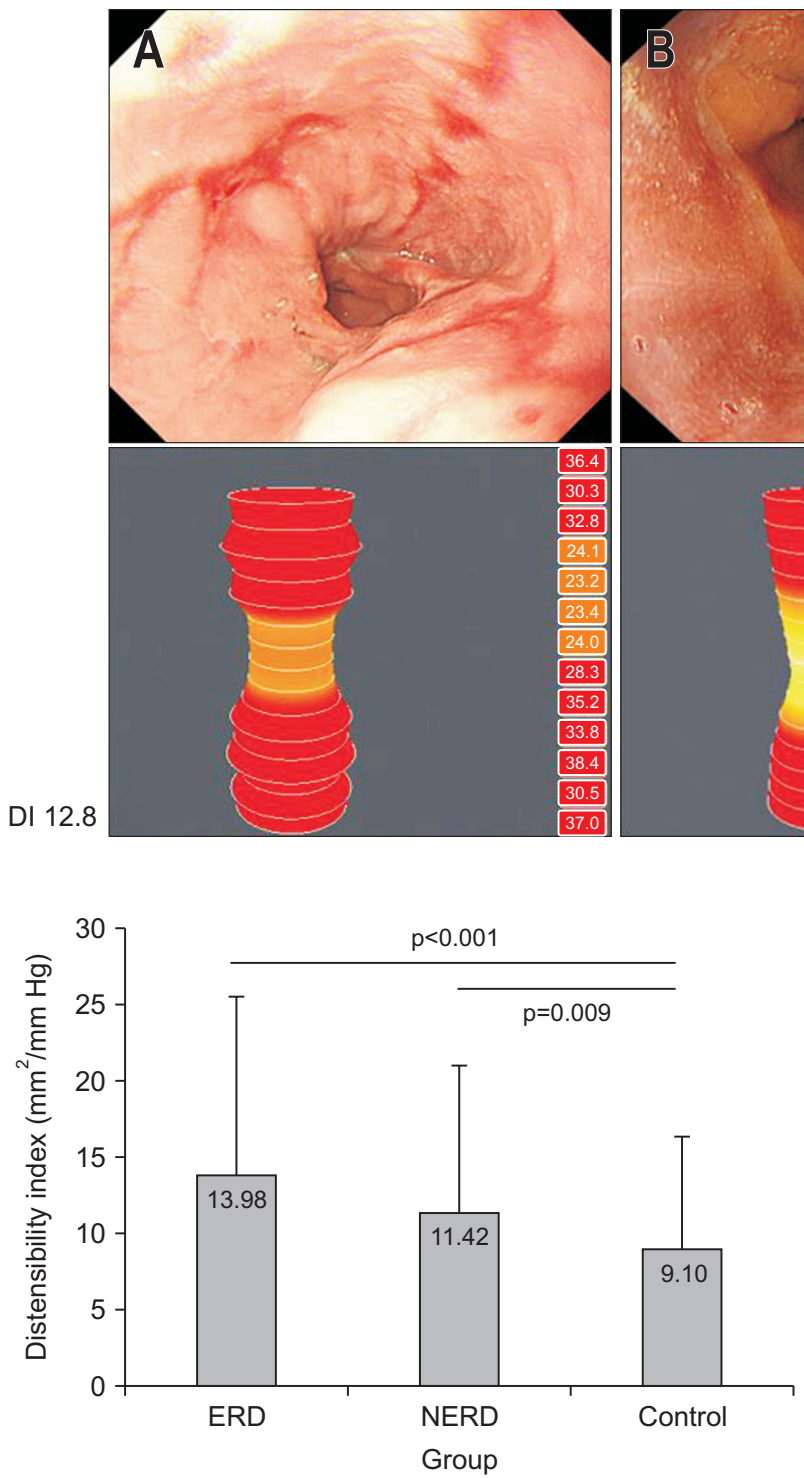

Fig. 3. Distensibility index for the gastroesophageal reflux disease group and controls. The distensibility index, measured by EndoFLIP ${ }^{\circledR}$, was significantly higher in the erosive reflux disease (ERD) group and nonerosive reflux disease (NERD) group than in the control group $(p<0.001)$

the EGJ for GERD diagnosis by receiver operating characteristic curve analysis; the area under the curve was 0.610 ( $95 \%$ confidence interval, 0.569 to $0.650 ; \mathrm{p}=0.021$ ) (Fig. 4). Considering the sensitivity (0.432) and specificity (0.743), we determined the distensibility index cutoff value to be 10.95 .

\section{DISCUSSION}

GERD is a disease that causes the reflux of gastric acid or food into the esophagus and leads to associated symp-
Fig. 2. Typical image of EndoFLIP ${ }^{\circledR}$ in erosive reflux disease $(A)$ and control patients (B).

DI 5.0 DI, distensibility index. toms. ${ }^{2,17}$ In Western Europe and North America, the proportion of individuals experiencing GERD at least once a week is as high as $20 \%$ to $30 \%$ of the population. ${ }^{3}$ GERD is classified as ERD or NERD without the presence of esophageal mucosal abnormalities, but with symptoms such as heartburn, ${ }^{18}$ and can progress to Barrett's esophagus if neglected. GERD is one of the main causes of diseases such as esophageal ulceration, esophageal stricture, and tumors, and it can rapidly decrease the quality of life of patients. ${ }^{19}$

EndoFLIP $^{\circledast}$ is a device that can be used to measure the function of the EGJ and help to diagnose many esophageal functional diseases, such as achalasia. ${ }^{11}$ It can measure the distensibility, compliance, diameter, and CSA of the EGJ in real time, and thus help in assessing the function of the EGJ. ${ }^{11,20}$ Although endoscopy, high-resolution manometry, and 24-hour ambulatory $\mathrm{pH}$ monitoring are all helpful in diagnosing GERD, they have limitations when it comes to measuring the function of the EGJ. ${ }^{8}$ Measuring the distensibility of the EGJ in accordance with the pathophysiology of increasing reflux when the distensibility of the EGJ increases in GERD patients can assist in the diagnosis of GERD. ${ }^{6,7}$ Unlike the barostat, this method does not require fluoroscopy and has the advantage of being able to assess images quickly in real-time.

However, the usefulness of EndoFLIP ${ }^{\circledR}$ in the diagnosis of GERD is controversial. ${ }^{8,11}$ Kwiatek et al. ${ }^{8}$ investigated the usefulness of EndoFLIP ${ }^{\circledast}$ in the diagnosis of GERD, confirming that CSA and distensibility were significant parameters. However, only a small number of individuals, 20 patients and 20 controls, were studied. On the other hand, Tucker et al. ${ }^{11}$ demonstrated that the use of EndoFLIP ${ }^{\circledast}$ to 
Table 2. Comparison of EndoFLIP ${ }^{\circledR}$ with High-Resolution Manometry and 24-Hour pH Ambulatory

\begin{tabular}{|c|c|c|c|c|}
\hline Characteristic & $\mathrm{ERD}(\mathrm{n}=204)$ & NERD $(n=310)$ & Control ( $n=277)$ & $\mathrm{p}$-value \\
\hline Distensibility index, $\mathrm{mm}^{2} / \mathrm{mm} \mathrm{Hg}$ & $13.98 \pm 11.54$ & $11.42 \pm 9.62$ & $9.10 \pm 7.23$ & $<0.001$ \\
\hline Cross-sectional area, $\mathrm{mm}^{2}$ & $291.03 \pm 160.77$ & $285.87 \pm 155.47$ & $249.78 \pm 144.76$ & 0.004 \\
\hline \multicolumn{5}{|l|}{ High-resolution manometry } \\
\hline IRP, $\mathrm{mm} \mathrm{Hg}$ & $10.19 \pm 6.84$ & $12.37 \pm 8.13$ & $12.64 \pm 7.92$ & 0.023 \\
\hline \multicolumn{5}{|l|}{ 24-Hour ambulatory $\mathrm{pH}$ monitoring } \\
\hline All reflux episodes & $56.36 \pm 39.41$ & $53.31 \pm 38.59$ & $41.31 \pm 24.01$ & 0.044 \\
\hline Acid reflux episodes & $19.72 \pm 21.60$ & $20.99 \pm 24.99$ & $14.57 \pm 14.00$ & 0.023 \\
\hline Nonacid reflux episodes & $35.25 \pm 28.66$ & $32.19 \pm 25.03$ & $26.93 \pm 17.73$ & 0.021 \\
\hline DeMeester score & $7.88 \pm 10.70$ & $7.38 \pm 13.59$ & $3.20 \pm 4.61$ & $<0.001$ \\
\hline
\end{tabular}

Data are presented as mean \pm SD.

ERD, erosive reflux disease; NERD, nonerosive reflux disease; IRP, integrated relaxation pressure.

Statistical significance between groups was tested by analysis of variance.

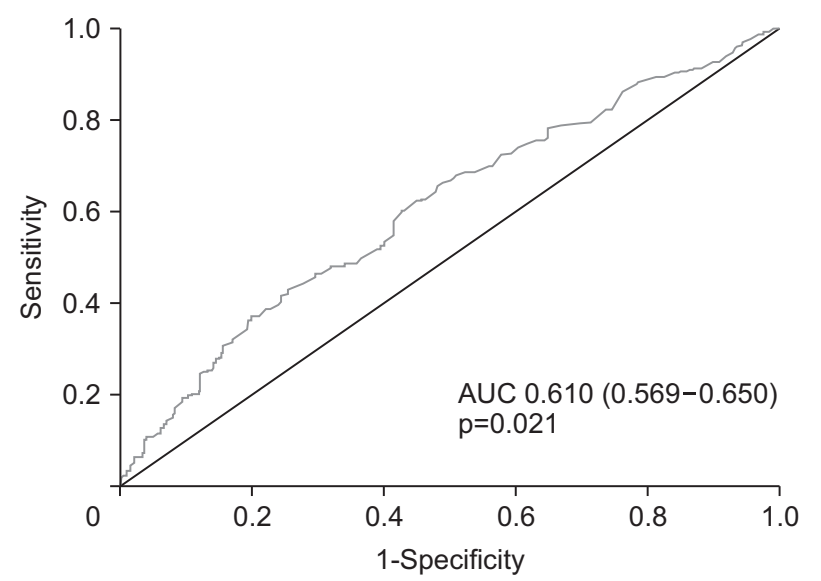

Fig. 4. Optimal distensibility index cutoff value. Receiver operating characteristic curve analysis showing the optimal distensibility index cutoff value. Cutoff value $=10.95$, with sensitivity $(0.432)$ and specificity (0.743).

AUC, area under the curve.

diagnose GERD was not significant in terms of CSA and distensibility, for a total of 40 subjects. Since then, there have been no large-scale studies on the usefulness of EndoFLIP $^{\oplus}$ in the diagnosis of GERD. Therefore, we conducted this study in order to evaluate the usefulness of EndoFLIP ${ }^{\circledR}$ in a large-scale population and to determine the differences between NERD and ERD using EndoFLIP ${ }^{\circledast}$.

In this study, we have shown that the distensibility index of the ERD, NERD, and control groups were significantly different. The ERD group had the highest distensibility index, while the control group had the smallest. In addition, the ERD group had the highest CSA, while the control group had the lowest. When comparing the results of highresolution manometry and 24-hour $\mathrm{pH}$ monitoring, the integrated relaxation pressure was lower in the ERD group than in the other groups, and 24-hour $\mathrm{pH}$ monitoring showed a significant difference in all groups. We demonstrated the usefulness of measuring EGJ distensibility in diagnosing GERD in a large-scale study of 791 patients. In the diagnosis of GERD through receiver operating characteristic curve analysis, it is important to obtain the distensibility index cutoff value. Furthermore, we have to consider the practical aspects of the EndoFLIP ${ }^{\circledast}$ technique, such as the assessment of the anti-reflux procedure for GERD patients, given that altered distensibility could predict worse symptoms or quality of life after the anti-reflux procedure. $^{21}$ In addition, because of expensive cost, EndoFLIP ${ }^{\circledast}$ may be not recommended to all GERD patients. It may be required in some cases, for example, before treatment such as anti-reflux mucosal resection or anti-reflux surgery, or in refractory GERD, as it is thought to be an additive diagnostic method when the information of distensibility is needed.

This study has several limitations. First, we did not investigate the presence of hiatal hernias. Hiatal hernias can alter the pressure when the low esophageal sphincter enters the thorax. It is important to control for the presence of hiatal hernias when measuring the distensibility index among the groups. However, hiatal hernias were difficult to objectify through endoscopic reviews. Second, we divided patients into ERD and NERD groups based on typical reflux symptoms which responded to proton pump inhibitors as in the previous study. ${ }^{8}$ By this definition, the NERD group could include reflux hypersensitivity. In this study, the mean values of the DeMeester score of the ERD and NERD groups was less than 14.7 points. We inferred that defining GERD patients based on symptoms might lead to false positive patients, whereas the DeMeester score can lead to highly false negative patients considering that the sensitivity of $\mathrm{pH}$ monitoring is $70 \%$ to $80 \% .^{12}$ When diagnosing based on symptoms, some GERD patients showed a low DeMeester score. ${ }^{11}$ Therefore, in this study, ERD group was defined according to typical reflux symptoms and endoscopic findings, and NERD was defined according to typical reflux symptoms, endoscopic findings and response 
to proton pump inhibitor. Control group was defined according to nonspecific abdominal symptoms, endoscopic findings and $\mathrm{pH}$ monitoring. The classification of ERD, NERD, and control group in this study may be confusing. Third, there is a relatively large variability among the patients, and, despite repeating the measurements thrice, the real-time recording may have led to variation during intraprocedural changes. However, since a significant difference was observed between the controls and the patients with GERD, we concluded that EndoFLIP ${ }^{\oplus}$ could provide additional information through the measurement of EGJ function. Fourth, in this study, we only used a $40 \mathrm{~mL}$ volume-controlled distension. Although there is no established volume distension during the EndoFLIP ${ }^{\circledast}$ procedure, $30-\mathrm{mL}$ and $40-\mathrm{mL}$ volumes appear to be the most clinically relevant based on previously published data. ${ }^{22}$ However, in our study, we standardized the volume to $40 \mathrm{~mL}$ and measured the parameters in a $40 \mathrm{~mL}$ volume distension, which may serve to reduce the bias among the study groups.

In conclusion, EGJ distensibility in GERD patients showed a significant increase compared to that of the control group, regardless of the presence of erosive esophagitis. In this large-scale study, the measurement of EGJ distensibility using the EndoFLIP ${ }^{\oplus}$ system was helpful in the diagnosis of GERD. In addition to ambulatory $\mathrm{pH}$-impedance tests, high-resolution manometry, and endoscopy, EndoFLIP $^{\oplus}$ is helpful in diagnosing GERD based on objective evidence and in classifying the subtype of GERD.

\section{CONFLICTS OF INTEREST}

No potential conflict of interest relevant to this article was reported.

\section{AUTHOR CONTRIBUTIONS}

Conceptualization: J.Y.C. Methodology: I.K.Y., E.K. Formal analysis: S.P.H. Writing - original draft: J.M.L. Writing - review and editing: I.K.Y. Approval of final manuscript: all authors.

\section{ORCID}

Jung Min Lee In Kyung Yoo Eunju Kim Sung Pyo Hong Joo Young Cho https://orcid.org/0000-0003-3551-8638 https://orcid.org/0000-0003-0909-339X https://orcid.org/0000-0002-3620-8175 https://orcid.org/0000-0001-6691-9069 https://orcid.org/0000-0002-7182-5806

\section{REFERENCES}

1. Hershcovici T, Jha LK, Fass R. Dexlansoprazole MR: a review. Ann Med 2011;43:366-374.

2. DeVault KR, Castell DO; American College of Gastroenterology. Updated guidelines for the diagnosis and treatment of gastroesophageal reflux disease. Am J Gastroenterol 2005; 100:190-200.

3. Dent J, El-Serag HB, Wallander MA, Johansson S. Epidemiology of gastro-oesophageal reflux disease: a systematic review. Gut 2005;54:710-717.

4. Kasamatsu S, Matsumura T, Ohta Y, et al. The effect of ineffective esophageal motility on gastroesophageal reflux disease. Digestion 2017;95:221-228.

5. Nathanson LK, Brunott N, Cavallucci D. Adult esophagogastric junction distensibility during general anesthesia assessed with an endoscopic functional luminal imaging probe (EndoFLIP $^{\circledR}$ ). Surg Endosc 2012;26:1051-1055.

6. Rinsma NF, Smeets FG, Bruls DW, et al. Effect of transoral incisionless fundoplication on reflux mechanisms. Surg Endosc 2014; 28:941-949.

7. Hoppo T, McMahon BP, Witteman BP, et al. Functional lumen imaging probe to assess geometric changes in the esophagogastric junction following endolumenal fundoplication. J Gastrointest Surg 2011;15:1112-1120.

8. Kwiatek MA, Kahrilas K, Soper NJ, et al. Esophagogastric junction distensibility after fundoplication assessed with a novel functional luminal imaging probe. J Gastrointest Surg 2010;14:268-276.

9. McMahon BP, Frøkjaer JB, Kunwald P, et al. The functional lumen imaging probe (FLIP) for evaluation of the esophagogastric junction. Am J Physiol Gastrointest Liver Physiol 2007;292:G377-G384.

10. Smeets FG, Keszthelyi D, Bouvy ND, Masclee AA, Conchillo JM. Does measurement of esophagogastric junction distensibility by EndoFLIP predict therapy-responsiveness to endoluminal fundoplication in patients with gastroesophageal reflux disease? J Neurogastroenterol Motil 2015;21:255-264.

11. Tucker E, Sweis R, Anggiansah A, et al. Measurement of esophago-gastric junction cross-sectional area and distensibility by an endolumenal functional lumen imaging probe for the diagnosis of gastro-esophageal reflux disease. Neurogastroenterol Motil 2013; 25:904-910.

12. El-Serag HB. Time trends of gastroesophageal reflux disease: a systematic review. Clin Gastroenterol Hepatol 2007;5:1726.

13. Regan J, Walshe M, Rommel N, Tack J, McMahon BP. New measures of upper esophageal sphincter distensibility and opening patterns during swallowing in healthy subjects using EndoFLIP(R). Neurogastroenterol Motil 2013;25:e25e34. 
14. Chen JW, Rubenstein JH. Esophagogastric junction distensibility assessed using the functional lumen imaging probe. World J Gastroenterol 2017;23:1289-1297.

15. DeHaan RK, Davila D, Frelich MJ, Gould JC. Esophagogastric junction distensibility is greater following Toupet compared to Nissen fundoplication. Surg Endosc 2017;31:193198.

16. Kessing BF, Bredenoord AJ, Schijven MP, et al. Long-term effects of anti-reflux surgery on the physiology of the esophagogastric junction. Surg Endosc 2015;29:3726-3732.

17. DeVault KR, Castell DO. Updated guidelines for the diagnosis and treatment of gastroesophageal reflux disease. Am J Gastroenterol 1999;94:1434-1442.

18. El-Serag H, Hill C, Jones R. Systematic review: the epidemiology of gastro-oesophageal reflux disease in primary care, using the UK General Practice Research Database. Aliment Pharmacol Ther 2009;29:470-480.

19. El-Serag HB, Sweet S, Winchester CC, Dent J. Update on the epidemiology of gastro-oesophageal reflux disease: a systematic review. Gut 2014;63:871-880.

20. Ponds FA, Bredenoord AJ, Kessing BF, Smout AJ. Esophagogastric junction distensibility identifies achalasia subgroup with manometrically normal esophagogastric junction relaxation. Neurogastroenterol Motil 2017;29:e12908.

21. Nabi Z, Reddy DN. Endoscopic management of gastroesophageal reflux disease: revisited. Clin Endosc 2016;49:408-416.

22. Ahuja NK, Agnihotri A, Lynch KL, et al. Esophageal distensibility measurement: impact on clinical management and procedure length. Dis Esophagus 2017;30:1-8. 